\title{
Trends in Medicine
}

\section{Case Report}

\section{Middle-aged male with purple skin}

\author{
Maricel Dela Cruz*, Marcus Greco and Molly Mulflur \\ Emergency Department, Aria Jefferson Health, Philadelphia, Pennsylvania, USA
}

\begin{abstract}
Disseminated intravascular coagulation (DIC) is a rare complication often associated with other concurrent medical diseases. Once DIC is diagnosed, rapid decline in clinical status can occur, leading to high morbidity and mortality. A well known historic cause of DIC includes severe sepsis secondary to gram-positive bacteria. Due to advances in medical care, more recent cases of fatality attributed to DIC associated with severe sepsis are rare. We present a case of 50-year-old male who experienced a devastating course of purpura fulminans after a progressive decline into septic shock as a result of influenza A pneumonia compounded with streptococcus bacteremia.
\end{abstract}

\section{Case description}

A 50-year-old male presented to the emergency department (ED) by ambulance with a one hour history of bluish-purple skin. The patient arrived from home, accompanied by his wife, who stated that two days prior, he complained of a headache, generalized body aches and chills. The patient developed a fever the following morning and spent the day in bed. As per emergency medical services, the patient was hypoxic and required supplemental oxygen in the field. In the ED, the patient's face, chest, and upper extremities appeared purple in color (Figures 1-3); he was tachycardic, hypotensive, and febrile.

\section{Diagnosis}

The patient was found to have disseminated intravascular coagulation (DIC) secondary to Influenza A pneumonia and associated Streptococcus bacteremia. Pictured is diffuse bodily and facial purpura with eyelid sparing. In particular, purpura fulminans is the presentation of diffuse ecchymotic skin lesions combined with fever and hypotension [1]. The patient's platelet count was $34,000 / \mu \mathrm{L}$

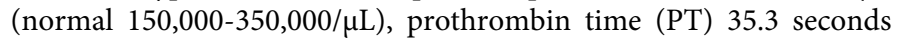

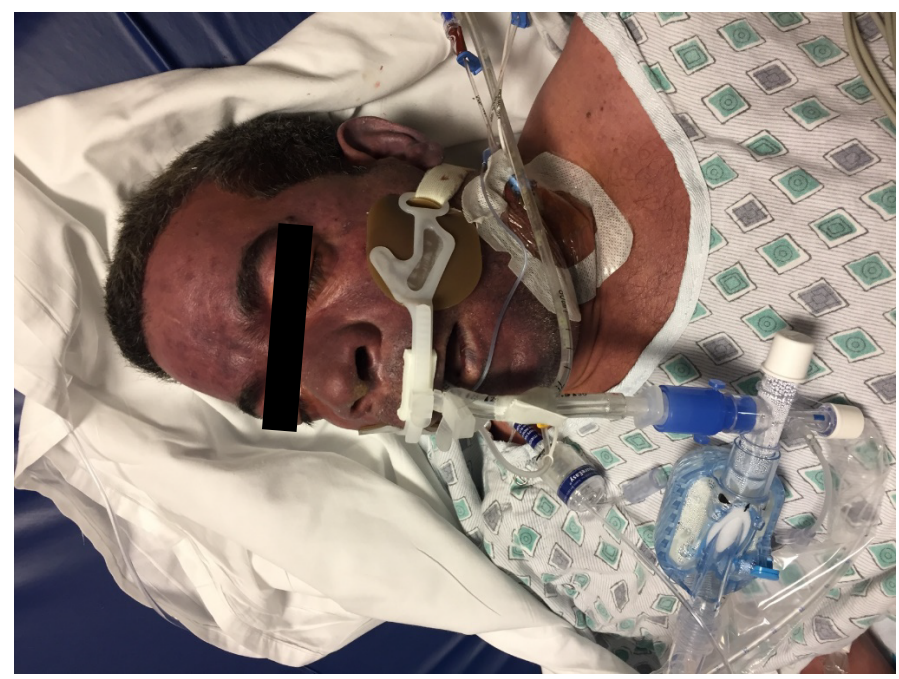

Figure 1. Image demonstrating facial purple skin color with eyelid sparing

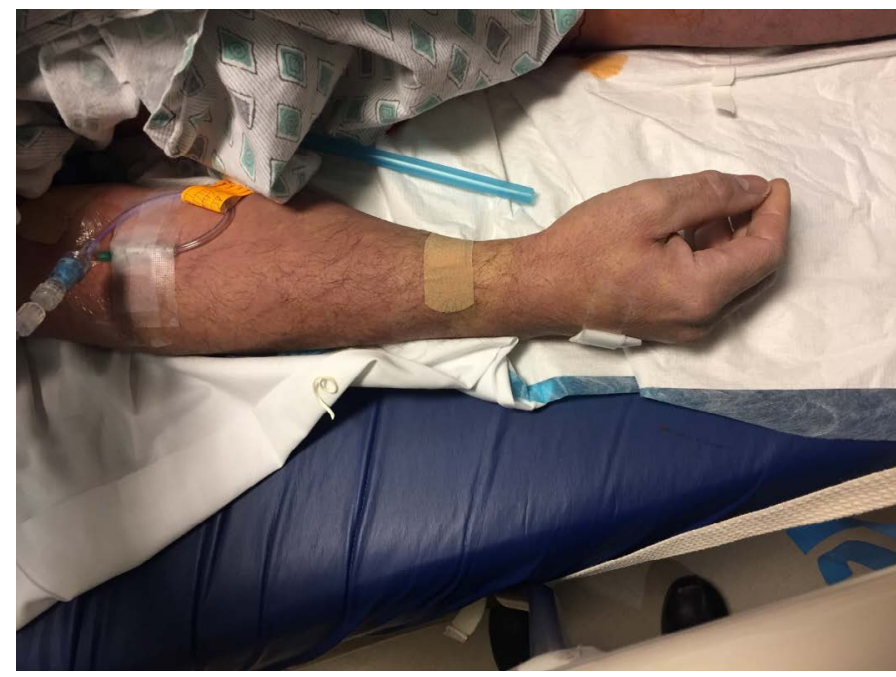

Figure 2. Upper extremity image displaying further ecchymotic skin lesions

(normal 9.0-12.5 seconds), partial thromboplastin time (PTT) 95 seconds (normal 22-38 seconds), D-Dimer $>5,000 \mathrm{ng} / \mathrm{mL}$ (normal $<230 \mathrm{ng} / \mathrm{mL}$ ), and fibrinogen $162 \mathrm{mg} / \mathrm{dL}$ (normal 182-532 mg/dL).

\section{Discussion}

DIC is often related to an underlying condition that causes a diffuse activation of clotting factors. The overuse of these factors then leads to depletion of such proteins and increased bleeding. Side effects include multiorgan failure and hemorrhage. Common lab findings include: a decreased platelet count on complete blood count (CBC), enlarged

Correspondence to: Maricel Dela Cruz, DO, MPH, Emergency Department, Aria Jefferson Health, Philadelphia, Pennsylvania, USA, E-mail: mdelacruz78@ gmail.com

Key words: disseminated intravascular coagulation, severe sepsis, purpura fulminans

Received: October 24, 2017; Accepted: November 10, 2017; Published: November 13, 2017 


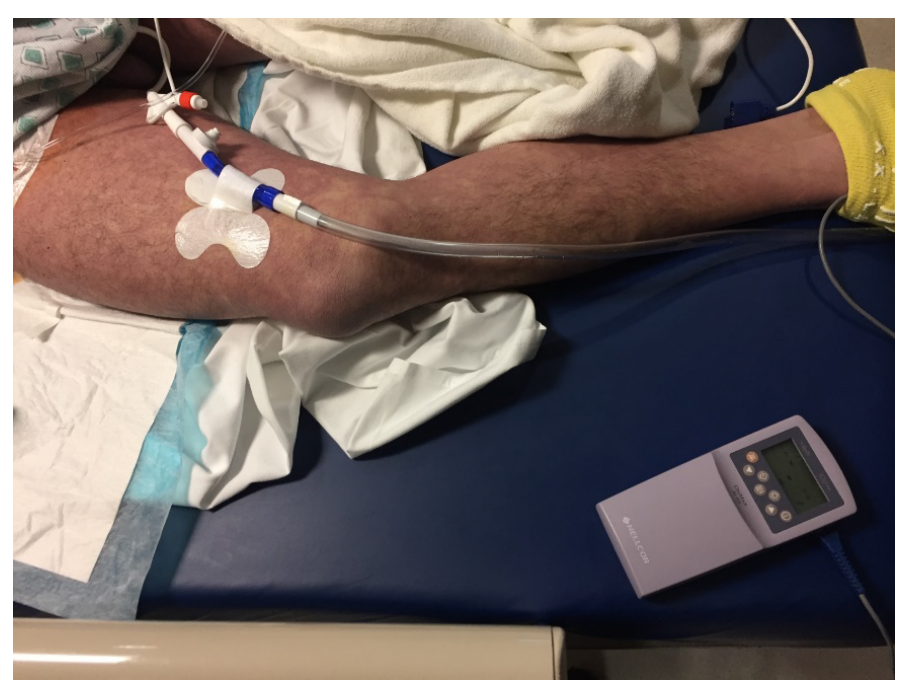

Figure 3. Lower extremity image exhibiting diffuse purple skin discoloration

platelets and fragmented red cells, or schistocytes, on peripheral blood smear, a prolonged PT, a prolonged PTT, a markedly elevated D-Dimer, and low fibrinogen level [2]. Treatment is aimed at determining the underlying cause, possible correction of platelets, restoring coagulation factors with fresh frozen plasma, and repletion of fibrinogen with cryoprecipitate to control severe bleeding as warranted [2].

DIC can be associated with sepsis, trauma, cancer, obstetrical complications, vascular disorders, and immunologic disorders.2 Coagulation is characterized by an increase in cytokine release, inflammation, and stimulation of fibrin and thrombotic occlusion of vessels. In patients with sepsis caused by either gram-negative or grampositive organisms, DIC may occur in $30-50 \%$ of untreated patients $[2,3]$. The likely clinical course of the patient pictured in this case was infection with Influenza A pneumonia and secondary streptococcal bacterial infection.

A historical example of non-bacterial infection and concurrent DIC includes the Spanish Flu epidemic in Spain during the 1910s. Similar to this patient, young and healthy individuals experienced a rapid progression of multiorgan failure, experiencing purpura fulminans immediately after being afflicted with influenza [4].

\section{Conclusion}

DIC may occur in $30-50 \%$ of untreated patients with sepsis caused by gram-positive organisms [2,3]. Treatment of DIC is supportive with identification of the underlying cause. Diffuse hemorrhage and multiorgan failure are severe complications. This patient was admitted to the intensive care unit, treated with intravenous antiviral medication and broad spectrum antibiotics, and unfortunately expired within two weeks of diagnosis. It is imperative to be aware of the rapid progression of DIC, as in the setting of sepsis and diffuse purpura mortality reaches almost 50\% without immediate diagnosis and treatment [5].

\section{References}

1. Carpenter CT, Kaiswer AB (1997) Purpura fulminans in pneumococcal sepsis: case report and review. Scand J Infect Dis 29: 479-483. [Crossref]

2. Levi M, Cate HT (1999) Disseminated intravascular coagulation. $N$ Engl J Med 341 586-592.

3. Van Gorp EC, Suharti C, Cate H, Dolmans WM, van der Meer JW, et al. (1999) Review: infectious diseases and coagulation disorders. J Infect Dis 180: 176-186. [Crossref]

4. Trilla A, Trilla G, Daer C (2008) The 1918 "Spanish Flu" in Spain. Clin Infect Dis 47: 668-673. [Crossref]

5. Lin SM, Wang YM, Lin HC, Lee KY, Huang CD, et al. (2008) Serum thrombomodulin level relates to the clinical course of disseminated intravascular coagulation, multiorgan dysfunction syndrome, and mortality in patients with sepsis. Crit Care Med 36: 683689. [Crossref]

Copyright: (C2017 Cruz MD. This is an open-access article distributed under the terms of the Creative Commons Attribution License, which permits unrestricted use, distribution, and reproduction in any medium, provided the original author and source are credited. 